\title{
BMJ Open Comparison of amblyopia in schoolchildren in Ireland and Northern Ireland: a population-based observational cross-sectional analysis of a treatable childhood visual deficit
}

\author{
Siofra Harrington, ${ }^{\oplus 1}$ Karen Breslin, ${ }^{2}$ Veronica O'Dwyer, ${ }^{1}$ Kathryn Saunders ${ }^{2}$
}

To cite: Harrington S, Breslin $\mathrm{K}$, O'Dwyer V, et al. Comparison of amblyopia in schoolchildren in Ireland and Northern Ireland: a population-based observational cross-sectional analysis of a treatable childhood visual deficit. BMJ Open 2019;9:e031066. doi:10.1136/ bmjopen-2019-031066

Received 13 April 2019 Revised 20 July 2019 Accepted 24 July 2019

\section{Check for updates}

(c) Author(s) (or their employer(s)) 2019. Re-use permitted under CC BY-NC. No commercial re-use. See rights and permissions. Published by BMJ.

${ }^{1}$ School of Physics and Clinical and Optometric Sciences,

Technological University Dublin, Dublin, Ireland

${ }^{2}$ School of Biomedical Sciences, Optometry and Vision Sciences, Ulster University, Coleraine, Northern Ireland, UK

Correspondence to Mrs Siofra Harrington; siofra.harrington@dit.ie

\section{ABSTRACT}

Objectives This study reports the prevalence of persistent amblyopia (post-traditional treatment age) in schoolchildren in the Republic of Ireland (henceforth Ireland) and Northern Ireland (NI), UK; populations with broadly similar refractive and genetic profiles but different eye-care systems.

Design This is a population-based observational study of amblyopia and refractive error.

Setting Recruitment and testing in primary and postprimary schools in Ireland and NI.

Participants Two groups identified through random cluster sampling to represent the underlying population; Ireland 898 participants (12-13 years old) and NI 723 participants (295 aged 9-10 years old, 428 aged 1516 years old).

Main outcome measures Monocular logMAR visual acuity (presenting and pinhole), refractive error (cycloplegic autorefraction), ocular alignment (cover test) and history of previous eye care. These metrics were used to determine prevalence and type of amblyopia and treatment histories.

Results Children examined in NI between 2009 and 2011 had a significantly lower amblyopia prevalence than children examined in Ireland between 2016 and 2018 (two-sample test of proportions, $p<0.001$ ). Using a criteria of pinhole acuity $0.2 \log M A R$ (6/9.5 Snellen) plus an amblyogenic factor, 4 of 295 participants aged $9-10$ years old $(1.3 \%, 95 \%$ Cls 0.4 to 3.6$)$ and 3 of 428 participants aged $15-16$ years old $(0.7 \%, 95 \%$ Cls 0.2 to 2.2) were identified in NI. The corresponding numbers in Ireland were 40 of 898 participants aged 12-13years old $(4.5 \%, 95 \% \mathrm{Cl} 3.2$ to 6.1$)$. In NI strabismic amblyopia was the most prevalent type of persistent amblyopia, whereas anisometropic was predominant in Ireland. In Ireland, amblyopia was associated with socioeconomic disadvantage $(\mathrm{OR}=2.2,95 \% \mathrm{Cls} 1.4$ to $3.6, \mathrm{p}=0.002)$ and poor spectacle compliance (OR $2.5,95 \%$ Cls 2.0 to 3.2 , $p<0.001)$.

Conclusions Amblyopia prevalence persisting beyond traditional treatment ages was significantly lower among NI children compared with Ireland. Uncorrected anisometropia, compliance with spectacle wear and socioeconomic disadvantage were contributing factors in Ireland. Children without obvious visible eye defects were

\section{Strengths and limitations of this study}

- This report is the first to profile and compare the prevalence of a treatable deficit of vision that develops in childhood (amblyopia) among children living in Ireland and Northern Ireland (NI), UK; two populations with similar refractive, demographic and genetic profiles but different public health and eye-care systems.

- An observational, population-based approach with random cluster sampling and comparable methodologies was used to obtain sufficiently large data sets from the two cohorts in Ireland and NI, allowing a direct comparison of prevalence.

- The randomly selected population-based recruitment protocols eliminate the potential bias associated with clinical samples.

- The participants in the Ireland (12-13years old) and NI (9-10 and 15-16years old) cohorts, while different age categories, were all older than the age range where treatment is effective (that is treatment before the age of 8 years).

- Pinhole vision was used as a surrogate for best-corrected vision.

less likely to access eye care in Ireland, resulting in missed opportunities for intervention where necessary.

\section{INTRODUCTION}

This paper reports on the first-ever amblyopia prevalence study in Ireland and compares findings with Northern Ireland (NI). Amblyopia, often referred to as 'lazy eye', is a relatively common, sight-threatening, but mostly treatable deficit of monocular, and occasionally, binocular vision. ${ }^{1}$ It results from anomalous visual experience during the critical period of visual development in the early years of childhood ${ }^{2}$ and may affect multiple aspects of visual function..$^{2-4}$ For example, untreated amblyopia does not self-rectify, ${ }^{5}$ impacts reading skills ${ }^{6}$ and significantly increases the 
risk of severe visual impairment of both eyes in later life. ${ }^{47}$ Amblyopia may affect an individual's quality of life, sports involvement, ${ }^{8} 9$ self-esteem, ${ }^{10}$ educational attainment ${ }^{4}$ and career choice. ${ }^{10}$ Annual lost earnings in the USA due to amblyopia have been estimated at $\$ 7$ billion. ${ }^{11}$ Due to the nature of the condition, treatment in early childhood (before the age of 8 years), ${ }^{12} 13$ when there is potential to improve vision, is essential. ${ }^{12-14}$

Amblyopia has a variety of causes: strabismic (ocular misalignment), anisometropic (a significant difference in refractive error between fellow eyes), form deprivation (exclusion of all visual information except light due to physical obstructions such as corneal/lenticular opacification or eyelid ptosis), significant uncorrected refractive error (high levels of astigmatism or hyperopia) or a combination of these features. ${ }^{115}$ Anisometropic amblyopia is asymptomatic in younger children, due to the clear image in the non-amblyopic eye, and this coupled with the lack of any obvious physical signs (straight eyes) can delay diagnosis ${ }^{416}$ and is associated with poorer compliance with treatment and spectacle wear. ${ }^{17}$ Although less common, high degrees of uncorrected refractive error in both eyes can result in bilateral (in both eyes) amblyopia. ${ }^{18}$

In Ireland and NI, publicly funded vision screening programmes to detect reduced vision are recommended at school entry. Conducting screening at this age ( $4-5$ years) balances the impetus for early identification of visual deficits in order to implement the most effective treatment with the need for children to cooperate with appropriately sensitive visual acuity tests. ${ }^{19}$ While school-entry vision screening recommendations and protocols are similar in both countries (table 1), there are important differences in terms of coverage, the postfailure pathway and the context in which the public health systems operate. Specifically, in NI all children are offered vision screening and children up to the age of 16 years (and up to 19 years if still in education) are entitled to free eye examinations and a voucher towards the cost of spectacles through self-referral to community (high street) optometry. Prompt referral to the hospital eye service for secondary care treatment can be initiated directly, with the cost of eye patches for occlusion therapy covered if required. In contrast, in order to obtain free eye care and a voucher towards the cost of spectacles in Ireland, children are referred into the public health system, where care is offered by ophthalmologists either in public hospital outpatients departments or in community healthcare clinics. The recent Health Service Executive review evaluating eye care services in Ireland identified concerns regarding capacity in public eye care services and the uniformity and reach of childhood vision screening. ${ }^{20}$

The purpose of this study is to report and compare the prevalence and cause of persistent amblyopia post-traditional treatment age $(8 \text { years })^{521}$ in two broadly similar population cohorts (in Ireland and $\mathrm{NI}$ ) with an equivalent genetic profile and similar refractive error prevalence, ${ }^{22}$ but different healthcare systems.

Table 1 A summary of public health service funded school-entry vision screening protocols and paediatric eye care services in Ireland and Northern Ireland

\begin{tabular}{|c|c|c|}
\hline Age & Ireland & Northern Ireland (UK) \\
\hline School entry $4-5$ years & $\begin{array}{l}\text { Monocular vision assessment at } 5 \text { years } \\
\text { (Snellen } 3 \mathrm{M} \text { chart or logMAR crowded } \\
3 \mathrm{M} \text { chart) is conducted by the school } \\
\text { nurse. } \\
\text { Pass: Vision of } 0.2 \text { logMAR in both eyes. } \\
\text { Fail: Vision worse than } 0.2 \text { logMAR in one } \\
\text { or both eyes or failure to complete the } \\
\text { test. } \\
\text { Failed vision screening referred to } \\
\text { hospital-based ophthalmology/ } \\
\text { community ophthalmology for follow-up. } \\
\text { Children remain in this system for review } \\
\text { until discharged at age } 12-16 \text { years. } \\
\text { Once an eye examination is carried out } \\
\text { in a hospital or community health clinic, } \\
\text { children under the age of } 12 \text { years can } \\
\text { apply for a voucher which will cover } \\
\text { the cost of basic frames and lenses. } \\
\text { Eye patches for occlusion therapy are } \\
\text { not provided free of charge; this cost is } \\
\text { covered by the parents. }\end{array}$ & $\begin{array}{l}\text { Monocular vision assessment at } 4-5 \text { years } \\
\text { (logMAR crowded } 3 \mathrm{M} \text { chart) is conducted by } \\
\text { the school nurse. } \\
\text { Pass: Vision of } 0.2 \text { logMAR in both eyes. } \\
\text { Fail: Vision worse than } 0.2 \text { logMAR in one or } \\
\text { both eyes or failure to complete the test. } \\
\text { Failed vision screening referred to hospital } \\
\text { eye service (orthoptist, optometrist, } \\
\text { ophthalmology) for follow-up. The } \\
\text { recommended maximum treatment duration } \\
\text { (treatment pathway) for refractive amblyopia } \\
\text { is } 38 \text { weeks and for strabismic amblyopia } 78 \\
\text { weeks; with children discharged to the care of } \\
\text { their community optometrist once treatment is } \\
\text { complete. } \\
\text { All children are entitled to a voucher which } \\
\text { covers the price basic spectacles and lenses. } \\
\text { The cost of eye patches for amblyopia } \\
\text { treatment is covered by the National Health } \\
\text { Service. }\end{array}$ \\
\hline Post-school entry & $\begin{array}{l}\text { If concerned, contact the general } \\
\text { practitioner or public health nurse for } \\
\text { a referral to a local ophthalmology } \\
\text { department. }\end{array}$ & $\begin{array}{l}\text { Attend high street optometrist (free of charge } \\
\text { for all children under } 16 \text { years and under } 19 \\
\text { years if still in education) and referred onto } \\
\text { tertiary care with ophthalmology if indicated. }\end{array}$ \\
\hline
\end{tabular}




\section{METHODS}

This study was a component of the Ireland Eye Study (IES) and the Northern Ireland Childhood Errors of Refraction (NICER) study, both observational population-based studies of visual status among schoolchildren in Ireland and NI. Ethical approval for the IES (20150305-Siofra Harrington, Ref 15-03) and NICER study (REC/05/121) was obtained from the Technological University Dublin Research Ethics Committee and the Ulster University Research Ethics Committee, respectively, with both studies adhering to the Tenets of Helsinki Declaration of Human Studies. Sampling, recruitment protocols and participation rates are previously published. ${ }^{22} 23$ Data collection took place between 2016 and 2018 in Ireland and between 2009 and 2011 in NI; best-corrected visual acuity (BCVA) data presented in the present study are from phase II (3-year follow-up) of the NICER study as this metric was not recorded in the initial phase of the NICER study.

In summary, stratified random sampling was employed in both studies to obtain representative samples of children in Ireland and NI schools. Schools were categorised by urban/rural status (urban: Ireland 751 of 898 participants (83.6\%), NI 414 of 723 participants (57.3\%); rural: Ireland 147 of 898 participants $(16.4 \%), 309$ of 723 participants $(42.7 \%)$ ) and socioeconomic status (disadvantaged: Ireland 108 of 898 participants (12.1\%), NI 337 of 723 participants (46.6\%); advantaged: 790 of 898 participants $(87.9 \%), 386$ of 723 participants $(53.4 \%))$. Ethnicity was assessed by the study coordinator and confirmed by the parent/guardian response in the study questionnaire. Both study populations were predominately white (NICER $98 \%$, IES $88.4 \%$ white and the remainder $11.6 \%$ were non-white (East Asian 3.3\%, South Asian 1.7\%, black 5.5\%, Arab 1.1\% combined)). Only data from participants at an age beyond which treatment is likely to be successful ( $>8$ years old $)^{12-14}$ were used in the present analysis. Participants were 898 participants aged 12-13 years living in Ireland and 723 participants (295 aged $9-10$ years and 428 aged $15-16$ years) living in NI.

\section{Procedures}

Children with written informed consent and child assent were examined on their school premises within school hours. Experimental techniques and methodology employed in both the NICER study and the IES have been described in detail elsewhere. ${ }^{22-24}$ In summary, both the NICER study and the IES examination involved:

1. Distance monocular crowded logMAR unaided and presenting (with spectacles if worn) visual acuities were measured and scored by-letter with and without a pinhole.

IES-using the Good-Lite (Elgin, Illinois, USA) Sloan letters $\log$ MAR chart. A light metre was employed to ensure the test luminance did not fall below $120 \mathrm{~cd} /$ $\mathrm{m}^{2}$; and the NICER study-logMAR chart on the Test Chart 2000, a Windows-based electronic test chart (http:/ / www.thomson-software-solutions.com/html/ test_chart_2000.html).

2. Ocular alignment was evaluated using a cover-uncover test and an alternating cover test using an accommodative target with and without spectacle correction (if worn) in the distance $(3 \mathrm{~m})$ and near $(40 \mathrm{~cm}$ IES and $33 \mathrm{~cm}$ NICER).

3. Cycloplegic autorefraction, at least $20 \mathrm{~min}$ postinstillation of anaesthetic (Minims Proxymetacaine Hydrochloride $0.5 \% \mathrm{w} / \mathrm{v}$, Bausch \& Lomb, UK) and cycloplegic eye drops (Minims Cyclopentolate Hydrochloride $1 \% \mathrm{w} / \mathrm{v}$, Bausch \& Lomb).

4. Direct dilated ophthalmoscopy examination of the anterior segment and the lens vitreous and fundus was carried out on all participants in Ireland and any abnormalities were noted.

5. Parents/legal guardians completed a participant and parental history and a children's lifestyle questionnaire detailing, inter alia, history of eye surgery, history of occlusion therapy and spectacle wear and whether participants had their eyes tested with an ophthalmologist or optometrist within 12 months before data collection.

6. Public involvement: the IES study questionnaire was based on the NICER study questionnaire with input from epidemiology, dietetics and focus group feedback. The questionnaire was refined following multisite user testing, which involved a cognitive walkthrough evaluation to assess the burden associated with and the time to complete the questionnaire. ${ }^{25}$ Following focus group feedback, a storyboard which outlined the IES examination was designed to make the study clear to children; the questionnaire was shortened by removing non-essential questions and simplifying the wording of the remaining questions to maximise accessibility; and a statement advising parents/guardians to skip any questions they felt uncomfortable answering was added to the document.

7. Follow-up: subsequent to the examination, all parents or legal guardians involved in both studies received a detailed report advising them of study findings and the necessity of any further treatment if required.

\section{Definitions}

To facilitate comparison with previous studies, amblyopia prevalence in this study was analysed and presented using two definitions:

Criteria (A) Multiethnic paediatric eye disease study (MEPEDS) definition: Unilateral amblyopia; $\geq 2$ line interocular difference in visual acuity, measured through a pinhole, with BCVA of 0.2logMAR (6/9.5 Snellen, $20 / 32$ ) or poorer in the worse eye in the presence of a unilateral amblyogenic factor consistent with the affected eye. Amblyogenic factors included strabismus at a distance and near fixation with/without spectacle correction, a history of strabismus surgery, anisometropia ( $\geq 1.00 \mathrm{D}$ difference in hyperopia, $\geq 3.00 \mathrm{D}$ difference in myopia, $\geq 1.50 \mathrm{D}$ difference in astigmatism in any meridian). ${ }^{26}$ 
Bilateral amblyopia: BCVA poorer than 0.3logMAR (6/12 Snellen, 20/40) in both eyes in the presence of a bilateral amblyogenic factor. ${ }^{26}$

Criteria (B) Refractive error study in children (RESC) definition: Amblyopia was defined as BCVA (measured through a pinhole) $\geq 0.3 \log$ MAR $(\leq 6 / 12$ Snellen, 20/40) in at least one eye associated with one or more of the following potential causes: (1) esotropia, exotropia or vertical tropia at $4 \mathrm{~m}$ fixation, or esotropia or vertical tropia at $0.5 \mathrm{~m}$ (strabismic amblyopia); (2) anisometropia of $\geq 2 \mathrm{D}$ spherical equivalent refractive error (SER) (anisometropic amblyopia); or (3) hyperopia of $\geq 6 \mathrm{D}$ SER.

Unilateral amblyopia: If only one eye met the criteria.

Bilateral amblyopia: If both eyes met the criteria separately. $^{27}$

Participants with a history of amblyopia treatment were not categorised as having persistent amblyopia unless they met criteria A or criteria B.

To examine the magnitude of amblyopic visual acuity deficits, based on the BCVA in the amblyopic eye, severe amblyopia was defined as BCVA $>0.6 \log$ MAR. ${ }^{28}$

All refractive errors were measured using autorefraction under cycloplegia and defined as follows: myopia SER $\leq-0.50$ dioptre sphere (DS), hyperopia SER $\geq+2.00$ DS and astigmatism $\geq 1.00$ dioptre cylinder $(\mathrm{DC}){ }^{22}$

\section{Statistical methodology}

The authors collected the primary data which were entered into databases which were used for subsequent analysis. The NICER study used Intercooled Stata V.9.2 (StataCorp, Texas, USA). The Statistical Package for Social Sciences (SPSS) V.24.0 was used for most analyses in the IES. The statistical programming language R, RStudio V.1.1.456 (R Foundation for Statistical Computing, Vienna, Austria), was used to generate random numbers for the sampling procedure, to provide prevalence data CIs and for comparing the prevalence of amblyopia, anisometropia, strabismus, spectacle wear and history of amblyopia treatment between the two populations, and to calculate the OR for amblyopia plus CIs. The sample sizes required ( $\mathrm{n}=716$ in both cohorts) were based on a predictive amblyopia prevalence of $1.9 \%$ (which included those previously treated for amblyopia), in predominately European Caucasian Australian 12 years old, ${ }^{29}$ with a 0.95 confidence level and a 0.01 precision estimate. The sample size was estimated as follows using Cochran's sample size formula ${ }^{30}: \mathrm{n}=$ sample size,

$\mathrm{Z}=1.96$ with confidence level $=0.95$ (for alpha $=0.05$ ), $\mathrm{d}=$ desired precision of estimate $=0.01$,

$\mathrm{P}=0.019$ (the estimated proportion)

$$
n=\frac{[(1.96)(0.019)(1-0.019)]}{(0.01)}
$$

Result: $\mathrm{n}=716.04$

The two-sample test for equality of proportions with continuity correction was used to compare prevalence between populations. Logistic regression analysis was used to examine the relationship between amblyopia prevalence and associated factors; participants without amblyopia were the reference category. The $5 \%$ level of significance was used throughout; CIs were $95 \%$.

\section{RESULTS}

Table 2 displays amblyopia prevalence for the right eye, left eye, either eye, unilateral and bilateral for both study groups (Ireland 12-13years old and NI 9-10and 15-16 years old) using criteria A and criteria B. All participants in NI were older than the critical treatment age and as there was no significant difference in amblyopia prevalence between the participants aged 9-10 and 15-16years old in NI using either criterion; unless otherwise stated, amblyopia prevalence refers to amblyopia prevalence in either eye for participants aged 9-16years old in NI and participants aged 12-13years old in Ireland using criteria A. Amblyopia prevalence was $4.5 \%$ (40 of 898 participants) within the Ireland study (IES). The corresponding result for the NI (NICER) study participants was 1.0\% (7 of 723 participants). Participants in Ireland were significantly more likely to be amblyopic than participants in NI, OR 4.8 (95\% CIs 2.1 to $10.7, \mathrm{p}<0.001$ ). Table 2 displays amblyopia prevalence in 898 Ireland participants aged 12-13 years (second column table 2), and 723 NI participants aged 9-16 years (final column table 2). Amblyopia prevalence is also presented for the two age cohorts in the NICER study: 9-10years old (first column table 2) and 15-16years old (third column table 2).

The study questionnaire completion rate was between 99.4\% (history of spectacle wear and when last examined by an ophthalmologist or optometrist) and $99.7 \%$ (history of eye surgery or amblyopia treatment). Using the parental questionnaire data, 24 of 40 amblyopic participants $(60 \%)$ in Ireland had not had their eyes examined by an eye-care practitioner during the 12 months before data collection.

Forty-five of 723 NI participants $(6.2 \%)$ reported a history of amblyopia treatment, and only 1 of 723 participants aged 9-10years old $(0.02 \%)$ remained amblyopic. In comparison, 68 of 898 participants $(7.6 \%)$ reported a history of amblyopia treatment in Ireland; however, of these, 24 of 898 participants $(2.7 \%)$ remained amblyopic; 10 of 898 (1.1\%) of which had BCVA poorer than $0.6 \log M A R$ in their amblyopic eye. While the incidence of prior amblyopia treatment did not differ between studies $(\mathrm{p}=0.61)$, successful treatment outcomes were significantly poorer in the Ireland study $(\mathrm{p}<0.001)$.

In Ireland, 12 of 898 participants $(1.3 \%)$ reported a history of strabismus surgery; 8 of $898(0.9 \%)$ of which were not categorised as having persistent amblyopia; a history of strabismus surgery was not associated with persistent amblyopia in Ireland $(p=0.25)$. All participants in Ireland with a history of eye surgery reported having attended an ophthalmologist or optometrist for review within 12 months of participation in the present study.

Table 3 presents the prevalence of anisometropia and strabismus in Ireland and NI studies, including 


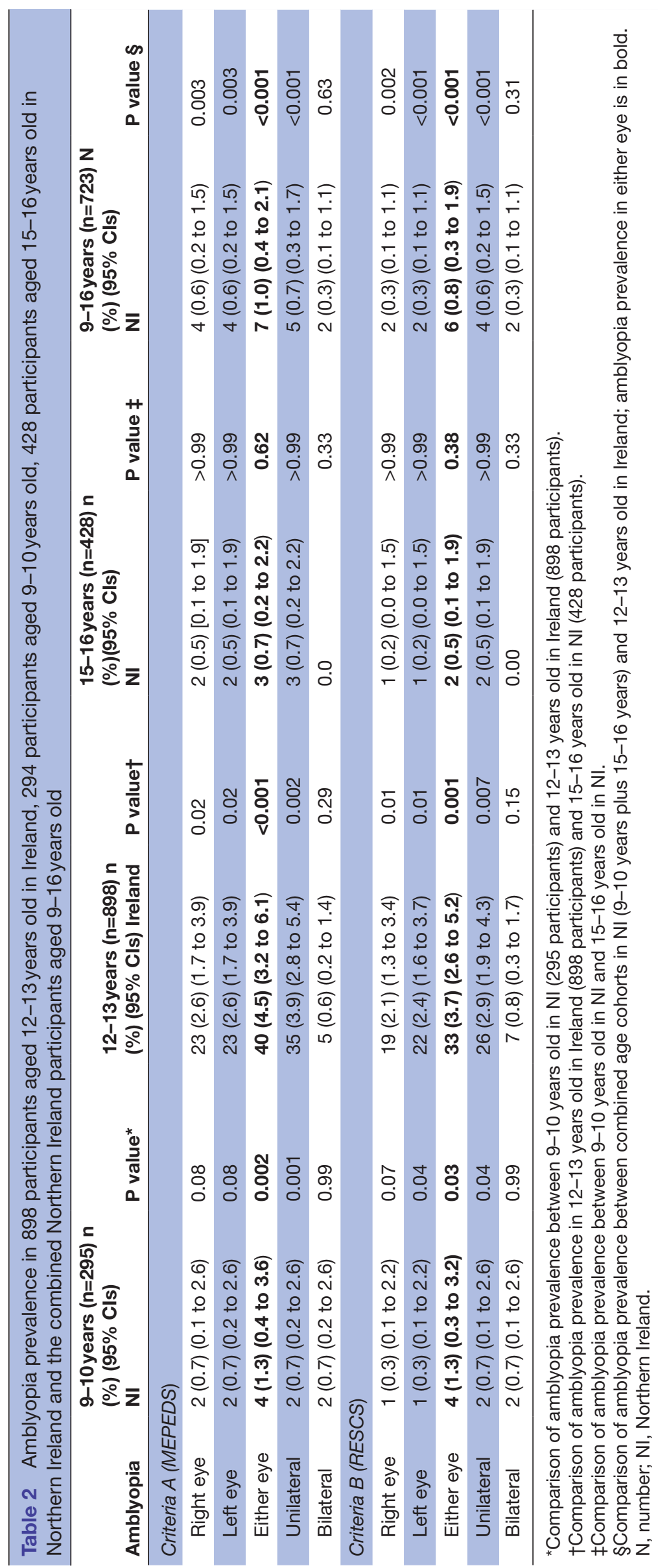


Table 3 Prevalence of anisometropia, strabismus and mixed mechanism anisometropia in Ireland (12-13 years) and NI (9-10 and $15-16$ years)

\begin{tabular}{|c|c|c|c|c|c|}
\hline $\begin{array}{l}\text { Anisometropia } \\
\text { strabismus status }\end{array}$ & $\begin{array}{l}\text { 9-10 years }(295) \\
\text { Prevalence }(\%, C / s) \\
\text { NI }\end{array}$ & P value* & $\begin{array}{l}\text { 12-13 years }(898) \\
\text { Prevalence }(\%, C l s) \\
\text { IRELAND }\end{array}$ & $\mathrm{P}$ valuet & $\begin{array}{l}15-16 \text { years }(428) \\
\text { Prevalence }(\%, \mathrm{Cls}) \\
\mathrm{NI}\end{array}$ \\
\hline Anisometropia & 26 (8.8) (6.0 to 12.8$)$ & 0.27 & $102(11.2)$ (9.4 to 13.7) & 0.59 & 43 (10.2) (7.4 to 13.4$)$ \\
\hline Strabismus & 17 (5.8) (3.5 to 9.2) & 0.35 & 38 (4.2) (3.1 to 5.8) & 0.68 & 21 (4.9) (3.1 to 7.5$)$ \\
\hline Anisometropia+strabismus & 4 (1.4) (0.5 to 3.9) & 0.99 & 13 (1.4) (0.8 to 2.5) & 0.61 & $4(0.1)(0.2$ to 3.8$)$ \\
\hline Strabismus only & $13(4.4)(2.6$ to 7.4$)$ & 0.24 & 25 (2.8) (1.8 to 4.1$)$ & 0.34 & 17 (4.0) (2.2 to 7.2$)$ \\
\hline
\end{tabular}

${ }^{*}$ Comparison of anisometropia, strabismus and mixed aetiology prevalence between Ireland (12-13 years old) and $\mathrm{NI}$ (9-10 years old). †Comparison of anisometropia, strabismus and mixed aetiology prevalence between the 15-16 years old in the NI study and 12-13 years old in Ireland.

$\mathrm{D}$, dioptre; N, number;NI, Northern Ireland.

participants with and without amblyopia. Table 4 presents the prevalence of hyperopia, myopia, astigmatism, anisometropia, strabismus and mixed aetiology (coexisting anisometropia plus strabismus) in the 898 participants aged 12-13years old in Ireland and the 723 participants aged 9-16years old in NI. There was no significant difference in the prevalence of either anisometropia or strabismus or mixed aetiology (coexisting anisometropia plus strabismus) between the participants in Ireland and NI (all p $>0.05$, see table 3 and table 4 ). There was no significant difference in the prevalence of astigmatism between the Ireland and NI participants. Figure 1 displays the prevalence of anisometropia, strabismus and mixed mechanism anisometropia plus strabismus in the 898 participants aged 12-13years old in Ireland and the 723 participants aged 9-16years old in NI.

\section{Causes of amblyopia}

For those participants classified as having amblyopia in the Ireland and NI studies, the reasons for amblyopia are illustrated in table 5 .

In Ireland, anisometropia was the most frequent cause of persistent amblyopia, followed by a combination of anisometropia and strabismus (mixed aetiology), strabismus only. In the NI study, strabismus was the primary cause of persistent amblyopia; only one participant had mixed aetiology, and one participant had anisometropia.

Level of amblyopic visual acuity Nine participants in Ireland had severe amblyopia (BCVA poorer than $0.6 \log \mathrm{MAR})$; all were anisometropic, three also had strabismus and all reported a history of amblyopia treatment. One NI participant aged 9-10years old had severe amblyopia; this participant had strabismus, hyperopia and was a bilateral hypermetropic astigmat with a history of amblyopia treatment.

Bilateral amblyopia In Ireland, five participants had bilateral amblyopia (criteria A): one had strabismus, one had mixed aetiology and the remainder refractive error (hypermetropic astigmatism). In the NI study, two participants $(0.3 \%)$ had bilateral amblyopia (aged 9-10years old); both were orthotropic with uncorrected refractive error (hypermetropic astigmatism). There were no NICER participants aged 15-16years old with bilateral amblyopia.

Spectacle wear The prevalence of spectacle wear was significantly higher $(\mathrm{p}<0.001)$ in NI (61 of 295 participants $(20.7 \%)$ aged $9-10$ years, and 146 of 428 participants $(34.1 \%)$ aged $15-16$ years) compared with Ireland (123 of 898 participants aged $12-13$ years $(13.8 \%)$ ). In

Table 4 Prevalence of refractive error, anisometropia, strabismus, and mixed aetiology in 898 participants aged 12-13years old in Ireland and 723 participants aged 9-16years old in NI

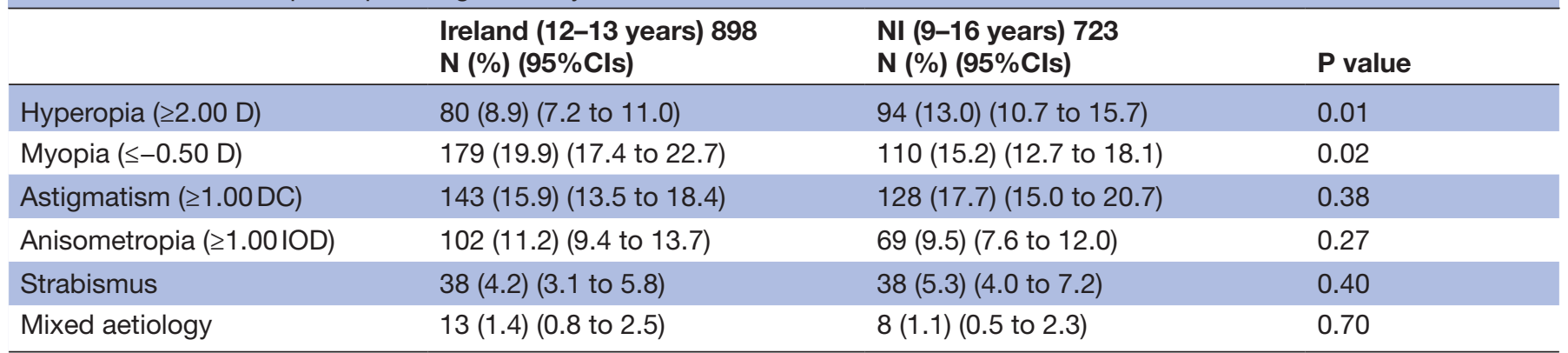

D, dioptre; DC, dioptre cylinder; IOD, interocular difference; N, number; NI, Northern Ireland. 


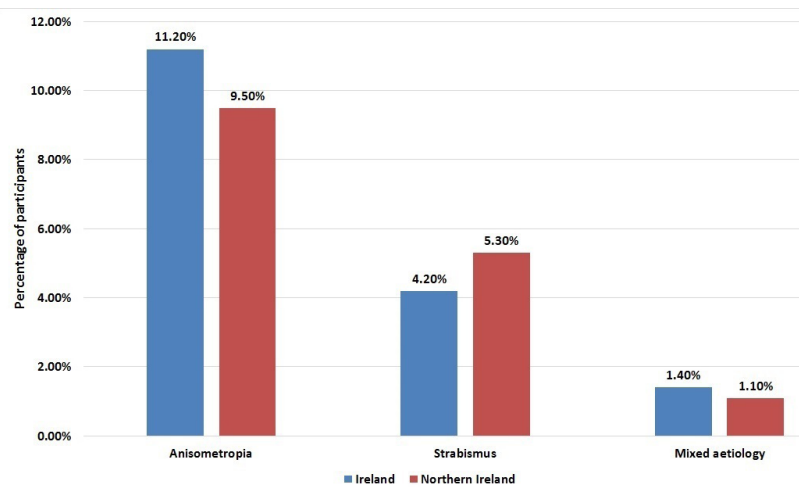

Figure 1 The prevalence of anisometropia, strabismus and mixed aetiology (coexisting strabismus and anisometropia) in 898 participants aged 12-13 years old in Ireland and 723 participants aged 9-16years old in Northern Ireland.

Ireland, a further 96 of 898 participants (10.7\%) did not have their spectacles in school.

In Ireland, 13 of the 40 amblyopic participants $(32.5 \%)$ never had spectacles, of which 1 had strabismus, and the remainder had uncorrected refractive error (anisometropia and hyperopic astigmatism). Ten of the 40 amblyopic participants $(25.0 \%)$ were wearing spectacles, 6 of which had strabismus and 4 had anisometropia. Seventeen of the 40 amblyopic participants $(42.5 \%)$ did not have their spectacles in school, 4 of which had strabismus and the remainder had anisometropia.

\section{Other factors associated with amblyopia}

Socioeconomic disadvantage was significantly associated with amblyopia in Ireland $(\mathrm{OR}=2.2,95 \%$ CIs 1.4 to 3.6 , $\mathrm{p}=0.002$ ). Amblyopia prevalence was $9.3 \%$ (10 of 108 participants) in socioeconomically disadvantaged participants compared with $3.8 \%$ (30 of 790 participants) in socioeconomically advantaged participants. There was a larger number of socioeconomically disadvantaged participants in NI (337 of 723 participants 46.6\%, compared with Ireland 108 of 898 participants 12\%); however, socioeconomic disadvantage was not associated with amblyopia in the NI study. It should be noted that the definition of disadvantaged in NI referred to a disadvantaged area where a school was located, ${ }^{24}$ whereas the definition of disadvantaged in Ireland referred to a threshold number of pupils in a school coming from a disadvantaged area. ${ }^{22}$

Amblyopia prevalence did not vary with ethnicity in Ireland (criteria A: white $4.7 \%$ (37 of 794 participants), non-white $2.9 \%$ (3 of 104 participants), $\mathrm{p}=0.41$, criteria B: white $3.8 \%$ (30 of 794 participants), non-white $2.9 \%$ (3 of 104 participants), $\mathrm{p}=0.65$ ). All NI participants were white.

Amblyopia was not associated with urban or rural living in Ireland or NI.

\section{DISCUSSION}

This report is the first to profile and compare the prevalence of amblyopia in children living in Ireland and NI; two populations with broadly similar refractive, demographic and genetic profiles but different public health and eye-care systems. Common methodology, including cycloplegic refraction, and definitions were applied in both cohorts, allowing a direct comparison of prevalence. Both cohorts sample size was sufficient to accurately determine amblyopia prevalence. Furthermore, the randomly selected population-based recruitment protocols eliminated the potential bias associated with clinical samples. The greater than $99 \%$ questionnaire completion rate was most likely due to public involvement in the design stage of the study. These novel data demonstrate that children living in Ireland are more likely to retain amblyopia into teenage years compared with children living in NI. Study findings ought to provide the prevalence estimates necessary to inform public healthcare policy on the resources required to address amblyopia. Data collection took place in 2009-2010 in the NICER study phase II and between 2016 and 2018 in Ireland. The impact of any material

Table 5 Causes of amblyopia in either eye for children in Ireland (12-13 years) and NI (9-10 years old, 15-16 and 9-16 years old combined)

\begin{tabular}{|c|c|c|c|c|}
\hline Ocular outcomes & $\begin{array}{l}\text { NI }(295) \\
\text { 9-10years n (\%) }\end{array}$ & $\begin{array}{l}\text { Ireland (898) } \\
\text { 12-13years n (\%) }\end{array}$ & $\begin{array}{l}\text { NI }(428) \\
15-16 \text { years n (\%) }\end{array}$ & $\begin{array}{l}\text { NI }(723) \\
9-16 \text { years n (\%) }\end{array}$ \\
\hline \multicolumn{5}{|l|}{ Criteria A (MEPEDS) } \\
\hline Strabismus & $2(50.0)$ & 5 (12.5) & $1(33.0)$ & $3(43.0)$ \\
\hline Mixed mechanism & $1(25.0)$ & $6(15.0)$ & $0(0.00)$ & $1(14.2)$ \\
\hline \multicolumn{5}{|l|}{ Criteria B (RESC) } \\
\hline Anisometropia & $1(25)$ & 19 (57.6) & $0(0.0)$ & $1(16.7)$ \\
\hline Strabismus & $2(50.0)$ & $3(9.1)$ & $1(50.0)$ & $3(50.0)$ \\
\hline Mixed mechanism & $1(25.0)$ & 5 (15.2) & $0(0.00)$ & $1(16.7)$ \\
\hline Refractive error & 0.0 & $6(21.2)$ & $1(50.0)$ & $1(16.7)$ \\
\hline
\end{tabular}

$\mathrm{NI}$, Northern Ireland; n, number. 
socioeconomical and educational changes during the intervening years should be considered; however, while there has been an increasing use of technology in education, household living standards have not changed materially over the last decade with less than $0.6 \%$ growth per annum in real wages (allowing for inflation) in Ireland (www.cso.ie) $^{31}$ and a $7 \%$ loss in real wages in the UK between 2006 and 2015 according to the International Labour Organisation. ${ }^{32}$

The prevalence of two key amblyogenic factors, strabismus and anisometropia, did not differ significantly between the two cohorts. However, the visual outcome of children with these conditions was markedly different in the two countries. Strabismic amblyopia was the most common type of amblyopia which persisted beyond the traditional treatment period in the NI group, whereas anisometropic amblyopia was the most common type of persistent amblyopia in Ireland. The relatively low prevalence of anisometropic amblyopia and the relatively higher level of spectacle ownership and compliance among the NI children compared with their counterparts in Ireland demonstrate that potential or manifest anisometropic amblyopes were successfully identified and treated with spectacles. Participation in NICER phase I may have led to a heightened awareness among the parents of that cohort group of the importance of spectacle compliance and compliance with amblyopia treatment. However, NICER phase I reported spectacle compliance of $76 \%$ in 6-7years old and $77 \%$ in 12-13years old, which was higher than the IES where spectacle compliance was $65 \%$ in 12-13 years old..$^{33}$ Therefore, spectacle compliance was higher in NI than Ireland in advance of NICER phase I. Furthermore, the older age cohort in NICER phase I of 12-13years old were older than the critical treatment period. ${ }^{12}{ }^{13}$ Also, there was no significant difference in amblyopia prevalence between the 9-10years old and 15-16years old in the NICER phase II. Previous studies demonstrated that anisometropic amblyopia is typically amenable to spectacle correction and that early implementation of refractive adaptation protocols is successful in resolving anisometropic amblyopia in the majority of children. ${ }^{18} 3435$

Early childhood vision screening protocols are designed to identify reduced vision and are one of the primary means by which anisometropia without associated strabismus is detected, and hence potential anisometropic amblyopes identified and offered treatment. ${ }^{19}$ Parents are less likely to seek eye care when children have no obvious signs or symptoms of visual impairment compared with parents of children who have manifest strabismus. ${ }^{16}$ However, early recognition of children with reduced vision at screening is only the first step in identifying and successfully treating amblyopia. Successful treatment of amblyopia is age-sensitive; so timely referral for diagnosis and treatment is key. ${ }^{12}$ The disparity between the primary aetiology of residual amblyopia in the Ireland and NI cohorts, with anisometropia being the primary aetiology associated with residual amblyopia in children in Ireland, may be indicative of inadequate vision screening coverage (Ireland $80 \%$, NI 95\%), ${ }^{36}$ and lack of timely access to eye care and spectacle correction and compliance with amblyopia treatment in Ireland. ${ }^{37}$ For example, amblyopia was associated with socioeconomic disadvantage in Ireland where the onus is on parents to fund the cost of eyepatches for amblyopia therapy; in contrast, this cost is funded by the state in NI.

Similar numbers of children in Ireland and NI had been previously treated for amblyopia. Approximately one-third (35.3\%, 24 of 68 participants) in Ireland with a history of amblyopia treatment presented with amblyopia, indicating poor compliance with treatment or that treatment is taking place too late ${ }^{38}$ Furthermore, 10 of those $(17 \%, 10$ of 68$)$ with a history of treatment presented with vision poorer than $0.6 \log$ MAR $(6 / 24$ Snellen) in one eye. In NI, only one child with a history of amblyopia treatment presented with reduced acuities. Delays of over 2 years between screening and treatment have been acknowledged in Ireland. ${ }^{20}$ While Clarke et al found a 1-year delay in commencing treatment does not adversely affect the visual outcomes of young children with small interocular acuity differences, the authors concluded early treatment is associated with better outcomes. ${ }^{39}$ Also, following the introduction of free preschool education in 2010, children in Ireland may be older commencing primary school than children in NI. ${ }^{40}$ Thus conducting vision screening in preschool, as practised in Denmark, ${ }^{41}$ ought to be considered.

Bilateral amblyopia was uncommon. The prevalence did not differ significantly between the two cohorts. Although the numbers were small, bilateral amblyopia has more severe consequences as vision is significantly impaired in both eyes (five children in Ireland and two in NI were below the legal minimum driving vision standard). All but one of the participants categorised as bilaterally amblyopic were orthotropic; hence, amblyopia may have been prevented with compliant spectacle wear alone. ${ }^{42}$

Even though anisometropia and strabismus are traditionally reported as the primary causes of amblyopia internationally, ${ }^{26}{ }^{27}$ uncorrected astigmatism is also a significant issue for participants both in NI and Ireland. Astigmatism prevalence in NI (6-7 years $24.0 \%, 12-13$ years $20.0 \%)$ and Ireland (6-7years $19.2 \%, 12-13$ years $15.9 \%$ ) is high, ${ }^{2243}$ and similar to other studies involving genetically isolated populations, it is significantly associated with persistent amblyopia in both cohorts. ${ }^{44}$ However, the low prevalence of persistent amblyopia found in NI participants in the present study demonstrates that early intervention and treatment can successfully reduce the risk of developing amblyopia in susceptible children.

In addition to quantifying a significant public health concern in Ireland, these data are a valuable addition to the limited literature on amblyopia prevalence, particularly the prevalence of residual amblyopia after treatment. Amblyopia prevalence rates in studies around the world vary depending on the definitions used and population studied. The RESC reported an overall amblyopia 
prevalence of $0.74 \%$, which ranged from $0.28 \%$ in African participants aged 5-15years old to $1.43 \%$ in Hispanic participants aged 5-15years old. ${ }^{27}$ In studies which involved Asian populations, amblyopia prevalence was $0.95 \%$ in the Chinese (predominately Han) aged 5-15years old in the $\operatorname{RESC}^{27}$ which was similar to that found in Chinese (Hani) aged 7-8years old $(0.97 \%)$, although slightly higher than that reported in Chinese (Hani) aged 13-14years old $(0.65 \%) .^{45}$ In contrast, a higher amblyopia prevalence was reported in UK participants aged 7 year olds $(3.6 \%)^{46}$ and Polish participants aged 3-12years old (3.1\%). ${ }^{47}$ Indeed comparison of the present study's findings to previous studies in other countries is not straightforward primarily because of the use of different definitions for amblyopia ${ }^{48}$ and variations in study methodology. For instance, similar to the Avon Longitudinal Study of Parents and Children (ALSPAC), ${ }^{49}$ pinhole acuity was used as a surrogate for BCVA, which facilitates comparison of study findings between Ireland and NI. A recent study demonstrated good agreement between pinhole acuity and BCVA and recommended it as a test for visual impairment not correctable with spectacles. This surrogacy ought to be considered when comparing results with studies where subjectively measured BCVA is reported. ${ }^{50}$ Furthermore, clarification is needed on whether reported amblyopia prevalence findings include figures for those previously treated for amblyopia. ${ }^{29}$ The present paper reports data relating to persistent amblyopia in older children after the critical period during which treatment is usually applied.

Amblyopia prevalence found in NI (9-16 years $1.0 \%)$ was significantly lower than in Ireland (12-13 years $4.5 \%)$ and also than other studies such as the ALSPAC ( 7 years $3.4 \%$ ) study $^{46}$ and the MEPEDS study in the USA (5-7 years Hispanic $3.1 \%$, Asian $1.1 \%) .{ }^{51}$ Amblyopia prevalence in NI was comparable to the Sydney Childhood Eye Study (12 years $0.4 \%$ ), which rose to $1.9 \%$ when participants previously treated for amblyopia were included and Denmark (11-12 years 1.5\%) where preschool vision screening has been in operation for 30 years. ${ }^{41}$ Furthermore, the Sydney-based study reported a reduction in amblyopia prevalence compared with the Blue Mountains study (also Australian), where $3.9 \%$ of the adult participants were diagnosed with amblyopia. ${ }^{2952}$ Similarly, amblyopia prevalence decreased from $2.9 \%$ in Danish adults ${ }^{53}$ to $1.5 \%$ following the initiation of the Danish preschool screening programme. ${ }^{41}$ Likewise, the present study reports a lower level of amblyopia in NI than that found 10 years previously in a retrospective study involving clinical data, where a $2.02 \%$ amblyopia prevalence was found in 8 years old in NI. ${ }^{54}$ In contrast, amblyopia prevalence in Ireland $(4.5 \%)$ was similar to that found in British military recruits $(4.4 \%)$ prior to the advent of comprehensive vision screening and amblyopia treatment in the UK. ${ }^{55}$ In addition, the Baltimore screening project found an amblyopia prevalence of $3.9 \%$ among socioeconomically disadvantaged inner-city children in Baltimore, ${ }^{56}$ with persistent amblyopia associated with poor compliance. ${ }^{57}$
Future research which investigates the reasons for poor spectacle compliance and poor treatment outcomes in Ireland when compared with NI is important. For example, longitudinal studies and audits of the present systems in Ireland and NI to examine the number of children referred for treatment and the proportion who attend for follow-up examination, the age at which amblyopia treatment and spectacles wear was initiated and the duration of and compliance with treatment are indicated in order to bring amblyopia prevalence in Ireland in line with NI. Electronic health records would facilitate analysis and audit of the present healthcare systems and allow trends to be monitored over time. ${ }^{58}$ Public eye health education directed at parents, teachers and the wider community is essential to promote spectacle and or amblyopia treatment compliance in vulnerable children.

\section{CONCLUSION}

This study is the first to report amblyopia prevalence in Ireland and the UK using robust methodologies and sampling to obtain a representative sample of children. The data reveal that school vision screening followed by prompt follow-up treatment results in successful outcomes in susceptible children, as evidenced by the UK participants in NI. The present study also reveals inequality in children's visual outcomes in two countries with an equivalent genetic profile and similar prevalence of amblyogenic factors but different public health systems and access to eye care. Children living in Ireland, where school-entry vision screening coverage is less comprehensive, free eye care less accessible and long waiting times exist, have a higher prevalence of amblyopia, which was associated with socioeconomic disadvantage. While most children in Ireland with visible eye defects were treated, uncorrected refractive error and poor spectacle compliance were critical issues. Future studies examining the for poor spectacle compliance in Ireland compared with NI are recommended. Consideration should be given to implementing changes, including public eye-care awareness programmes aimed at bringing the visual outcomes of children in Ireland in line with children in NI.

Acknowledgements The authors would like to express their gratitude for their valuable contributions to this research to Dr Lisa O'Donoghue and Dr Julie McClelland (Northern Ireland Childhood Errors of Refraction (NICER) study, School of Biomedical Sciences, University of Ulster, Co. Londonderry, Northern Ireland); Dr Jim Stack (Waterford Institute of Technology) and Professor John Kearney (Epidemiology, School of Biological Sciences, Technological University Dublin, Ireland) for their valuable input in the Ireland Eye Study (IES). Also, the authors would like to acknowledge the support and participation of the schools, the children, and their parents and guardians in the NICER study and the IES. The part played by the focus group participants in the design stage of the IES is also acknowledged. This work was supported by the College of Optometrists, UK, the Technological University Dublin Fiosraigh grant, the Opticians Board and the Association of Optometrists Ireland. The part played by the focus group participants in the design stage of the IES is also acknowledged.

Contributors All authors (SH, KB, VO'D and KJS) contributed to the conception and design of this work. All authors were involved in drafting, critically reviewing and approval of the final version to be published. 
Funding This work was supported by the College of Optometrists, UK, the Technological University Dublin Fiosraigh grant, the Opticians Board, and the Association of Optometrists Ireland

Disclaimer SH reports grants from Irish Opticians Board, grants from Association of Optometrists Ireland and grants from British College of Optometrists during the conduct of the study.

Competing interests The authors have read and understood British Medical Journal policy on declaration of interests and SH reports grants from Irish Opticians Board, grants from Association of Optometrists Ireland and grants from British College of Optometrists during the conduct of the study.

Patient and public involvement statement The study was supported by a patient advisory group which provided input to the programme of research. Parents/ legal guardians of participants partnered with us for the design of the study, the informational material to support the data collection and participant and school involvement, and assess the burden of participation from the patient's perspective. At the end of the study, results and findings were provided to all participants.

Patient consent for publication Not required.

Provenance and peer review Not commissioned; externally peer reviewed.

Data availability statement № data are available.

Open access This is an open access article distributed in accordance with the Creative Commons Attribution Non Commercial (CC BY-NC 4.0) license, which permits others to distribute, remix, adapt, build upon this work non-commercially, and license their derivative works on different terms, provided the original work is properly cited, appropriate credit is given, any changes made indicated, and the use is non-commercial. See: http://creativecommons.org/licenses/by-nc/4.0/.

\section{REFERENCES}

1. Holmes JM, Clarke MP, Amblyopia CMP. Amblyopia. The Lancet 2006;367:1343-51.

2. Webber AL, Wood J. Amblyopia: prevalence, natural history, functional effects and treatment. Clin Exp Optom 2005;88:365-75.

3. Meier K, Giaschi D. Unilateral amblyopia affects two eyes: fellow eye deficits in amblyopia. Invest. Ophthalmol. Vis. Sci. 2017;58.

4. Chua B, Mitchell P. Consequences of amblyopia on education, occupation, and long term vision loss. $\mathrm{Br} J$ Ophthalmol 2004;88:1119-21.

5. Holmes JM, Lazar EL, Melia BM, et al. Effect of age on response to amblyopia treatment in children. Arch Ophthalmol 2011;129:1451.

6. Kelly KR, Jost RM, De La Cruz A, et al. Amblyopic children read more slowly than controls under natural, binocular reading conditions. J Aapos 2015;19:515-20.

7. Rahi JS, Logan S, Timms C, et al. Risk, causes, and outcomes of visual impairment after loss of vision in the non-amblyopic eye: a population-based study. The Lancet 2002;360:597-602.

8. Niechwiej-Szwedo E, Goltz HC, Chandrakumar M, et al. Effects of anisometropic amblyopia on visuomotor behavior, I: saccadic eye movements. Invest Ophthalmol Vis Sci 2010;51.

9. Khalaj M, Zeidi IM, Gasemi MR, et al. The effect of amblyopia on educational activities of students aged 9 - 15. J Biomed Sci Eng 2011;04:516-21.

10. Carlton J, Kaltenthaler E. Amblyopia and quality of life: a systematic review. Eye 2011;25:403-13.

11. Membreno JH, Brown MM, Brown GC, et al. A cost-utility analysis of therapy for amblyopia. Ophthalmology 2002;109:2265-71.

12. Fronius $M$, Cirina L, Ackermann $\mathrm{H}$, et al. Efficiency of electronically monitored amblyopia treatment between 5 and 16 years of age: new insight into declining susceptibility of the visual system. Vision Res 2014;103:11-19.

13. Holmes JM, Levi DM. Treatment of amblyopia as a function of age. Vis Neurosci 2018;35:E015.

14. Koo EB, Gilbert AL, VanderVeen DK. Treatment of amblyopia and amblyopia risk factors based on current evidence. Semin Ophthalmol 2017;32:1-7.

15. Barrett BT, Bradley A, Candy TR. The relationship between anisometropia and amblyopia. Prog Retin Eye Res 2013;36:120-58.

16. Weakley DR. The association between nonstrabismic anisometropia, amblyopia, and subnormal binocularity. Ophthalmology 2001;108:163-71.

17. Bruce A, Sanders T, Sheldon TA. Qualitative study investigating the perceptions of parents of children who failed vision screening at the age of 4-5 years. Bmjpo 2018;2:e000307.
18. Tailor V, Bossi M, Greenwood JA, et al. Childhood amblyopia: current management and new trends. Br Med Bull 2016;119:75-86.

19. Carlton J, Karnon J, Czoski-Murray C, et al. The clinical effectiveness and cost-effectiveness of screening programmes for amblyopia and strabismus in children up to the age of 4-5 years: a systematic review and economic evaluation. Health Technol Assess 2008;12:iii, xi-194-194.

20. Murphy $B$, Health SE. Primary care eye services review group report; 2017: 1-108. file://C:/Users/302491/Downloads/Eye Review Report - Final.pdf.

21. Powell C, Hatt SR, Cochrane Eyes and Vision Group. Vision screening for amblyopia in childhood. Cochrane Database Syst Rev 2009;89.

22. Harrington SC, Stack J, Saunders K, et al. Refractive error and visual impairment in Ireland schoolchildren. Br J Ophthalmol 2018. doi:10.1136/bjophthalmol-2018-312573. [Epub ahead of print: 12 Oct 2018].

23. Breslin KMM, O'Donoghue L, Saunders KJ, et al. A prospective study of spherical refractive error and ocular components among Northern Irish schoolchildren (the NICER study). Invest Ophthalmol Vis Sci 2013;54:4843-50.

24. O'Donoghue L, Saunders KJ, McClelland JF, et al. Sampling and measurement methods for a study of childhood refractive error in a UK population. Br J Ophthalmol 2010;94:1150-4.

25. Collins D. Pretesting survey instruments: an overview of cognitive methods. Qual Life Res 2003;12:229-38.

26. Varma R, Deneen J, Cotter S, et al. The multi-ethnic pediatric eye disease study: design and methods. Ophthalmic Epidemiol 2006;13:253-62.

27. Xiao O, Morgan IG, Ellwein LB, et al. Prevalence of amblyopia in school-aged children and variations by age, gender, and ethnicity in a Multi-Country refractive error study. Ophthalmology 2015;122:1924-31

28. Wallace DK, Repka MX, Lee KA, et al. Amblyopia preferred practice Pattern®. Ophthalmology 2018;125:P105-P142.

29. Robaei D, Kifley A, Rose KA, et al. Impact of amblyopia on vision at age 12 years: findings from a population-based study. Eye 2008;22:496-502.

30. Daniel WW, Cross CL. Biostatistics : A Foundation for Analysis in the Health Sciences.. In: Daniel W, ed. 7Th. New York, 1999John Wiley \& Sons;. (Accessed May 13, 2019).

31. CPI Inflation Calculator, CSO - Central Statistics Office. Available: https://www.cso.ie/en/interactivezone/visualisationtools/cpiinflation calculator/ [Accessed May 18, 2019].

32. Ryder G. Global Wage Report 2016 / 17 Wage Inequality in the Workplace, 2016. Available: https://www.ilo.org/wcmsp5/groups/ public/-dgreports/-dcomm/-publ/documents/publication/wcms 537846.pdf [Accessed May 18, 2019]

33. O'Donoghue L, McClelland JF, Logan NS, et al. Refractive error and visual impairment in school children in Northern Ireland. $\mathrm{Br} J$ Ophthalmol 2010;94:1155-9.

34. Stewart CE, Moseley MJ, Fielder AR, et al. Refractive adaptation in amblyopia: quantification of effect and implications for practice. $\mathrm{Br} J$ Ophthalmol 2004;88:1552-6.

35. Chen P-L, Chen J-T, Tai M-C, et al. Anisometropic amblyopia treated with spectacle correction alone: possible factors predicting success and time to start patching. Am J Ophthalmol 2007;143:54-60.

36. Sloot F, Hoeve HLJ, de Kroon MLA, et al. Inventory of current EU paediatric vision and hearing screening programmes. J Med Screen 2015;22:55-64

37. Power W, Barry P, Moriarty P, et al. Clinical strategy and programmes DIRECTORATE patient safety first Tús Áite do Shábháilteacht Othar national clinical programme for ophthalmology model of eye care, 2017. Available: https://www.hse.ie/eng/services/publications/ clinical-strategy-and-programmes/model-of-eye-care.pdf [Accessed October 29, 2018]

38. Wang J. Compliance and patching and atropine amblyopia treatments. Vision Res 2015;114:31-40.

39. Clarke MPet al. Randomised controlled trial of treatment of unilateral visual impairment detected at preschool vision screening. BMJ 2003;327.

40. Murray A, McNamara E, Williams J, et al. Growing Up in Ireland THE LIVES OF 5-YEAR-OLDS. Dublin: ESRI, 2019.

41. Hansen $M H$, Munch IC, Li XQ, et al. Visual acuity and amblyopia prevalence in 11- to 12-year-old Danish children from the Copenhagen child cohort 2000. Acta Ophthalmol 2019;97:29-35.

42. Wallace DK, Chandler DL, Beck RW, et al. Treatment of bilateral refractive amblyopia in children three to less than 10 years of age. Am J Ophthalmol 2007;144:487-96. 
43. O'Donoghue L, Rudnicka AR, McClelland JF, et al. Refractive and corneal astigmatism in white school children in Northern Ireland. Invest Ophthalmol Vis Sci 2011;52:4048-53.

44. Dobson V, Miller JM, Harvey EM, et al. Amblyopia in astigmatic preschool children. Vision Res 2003;43:1081-90.

45. Zhu H, Pan C, Sun Q, et al. Prevalence of amblyopia and strabismus in Hani school children in rural Southwest China: a cross-sectional study. BMJ Open 2019;9:e025441.

46. Williams $\mathrm{C}$, Northstone K, Howard M, et al. Prevalence and risk factors for common vision problems in children: data from the ALSPAC study. Br J Ophthalmol 2008;92:959-64.

47. Polling J-R, Loudon SE, Klaver CCW. Prevalence of amblyopia and refractive errors in an unscreened population of children. Optom Vis Sci 2012;89.

48. Ohlsson J, Villarreal G, Sjöström A, et al. Visual acuity, residual amblyopia and ocular pathology in a screened population of 12-13-year-old children in Sweden. Acta Ophthalmol Scand 2001;79:589-95.

49. Williams C, Northstone K, Harrad RA, et al. Amblyopia treatment outcomes after preschool screening $\mathrm{V}$ school entry screening: observational data from a prospective cohort study. Br J Ophthalmol 2003;87:988-93.
50. Kumar RS, Rackenchath MV, Sathidevi AV, et al. Accuracy of pinhole visual acuity at an urban Indian Hospital. Eye 2019;33:335-7.

51. McKean-Cowdin R, Cotter SA, Tarczy-Hornoch K, et al. Prevalence of amblyopia or strabismus in Asian and non-Hispanic white preschool children: multi-ethnic pediatric eye disease study. Ophthalmology 2013;120:2117-24.

52. Attebo K, Mitchell P, Cumming R, et al. Prevalence and causes of amblyopia in an adult population. Ophthalmology 1998;105:154-9.

53. Vinding T, Gregersen E, Jensen A, et al. Prevalence of amblyopia in old people without previous screening and treatment. Acta Ophthalmol 1991;69:796-8.

54. Donnelly UM, Stewart NM, Hollinger M. Prevalence and outcomes of childhood visual disorders. Ophthalmic Epidemiol 2005;12:243-50.

55. Hopkisson B, Clarke JR, Oelman BJ. Residual amblyopia in recruits to the British Army. Br Med J 1982;285:940.

56. Preslan MW, Novak A. Baltimore vision screening project. phase 2. Ophthalmology 1998;105:150-3.

57. Simons K, Preslan M. Natural history of amblyopia untreated owing to lack of compliance. Br J Ophthalmol 1999;83:582-7.

58. Hartmann EE, Block SS, Wallace DK, et al. Vision and eye health in children 36 to <72 months: proposed data system. Optom Vis Sci 2015;92:24-30. 